\title{
Immune checkpoint inhibitor-associated myocarditis: manifestations and mechanisms
}

\author{
Javid Moslehi, ${ }^{1}$ Andrew H. Lichtman, ${ }^{2}$ Arlene H. Sharpe, ${ }^{3,4}$ Lorenzo Galluzzi, ${ }^{5,6,7}$ and Richard N. Kitsis ${ }^{8}$ \\ 'Division of Cardiovascular Medicine and Division of Oncology, Cardio-Oncology Program, Department of Medicine, Vanderbilt University Medical Center, Nashville, Tennessee, USA. ²Department of Pathology, \\ Brigham and Women's Hospital and Harvard Medical School, Boston, Massachusetts, USA. ${ }^{3}$ Department of Immunology and Blavatnik Institute, Harvard Medical School, Boston, Massachusetts, USA. \\ ${ }^{4}$ Evergrande Center for Immunologic Diseases, Harvard Medical School and Brigham and Women's Hospital, Boston, Massachusetts, USA. ${ }^{5}$ Department of Radiation Oncology, Sandra and Edward Meyer \\ Cancer Center, Caryl and Israel Englander Institute for Precision Medicine, Weill Cornell Medical College, New York, New York, USA. ${ }^{6}$ Department of Dermatology, Yale School of Medicine, New Haven, \\ Connecticut, USA. ${ }^{~ U n i v e r s i t e ́ ~ d e ~ P a r i s, ~ P a r i s, ~ F r a n c e . ~}{ }^{8}$ Departments of Medicine and Cell Biology, Wilf Family Cardiovascular Research Institute, and Albert Einstein Cancer Center, Albert Einstein College of \\ Medicine, Bronx, New York, USA.
}

\begin{abstract}
Immune checkpoint inhibitors (ICls) have transformed the treatment of various cancers, including malignancies once considered untreatable. These agents, however, are associated with inflammation and tissue damage in multiple organs. Myocarditis has emerged as a serious ICI-associated toxicity, because, while seemingly infrequent, it is often fulminant and lethal. The underlying basis of ICl-associated myocarditis is not completely understood. While the importance of T cells is clear, the inciting antigens, why they are recognized, and the mechanisms leading to cardiac cell injury remain poorly characterized. These issues underscore the need for basic and clinical studies to define pathogenesis, identify predictive biomarkers, improve diagnostic strategies, and develop effective treatments. An improved understanding of ICl-associated myocarditis will provide insights into the equilibrium between the immune and cardiovascular systems.
\end{abstract}

\section{Introduction}

Immune checkpoint inhibitors (ICIs) have emerged as highly effective therapies for many cancers $(1,2)$. Thus far, all approved ICIs are monoclonal antibodies that block cytotoxic T lymphocyte-associated protein 4 (CTLA-4), programmed cell death protein-1 (PD-1), or programmed death-ligand 1 (PD-L1), each a key inhibitor of T cell activation and function $(3,4)$. About $50 \%$ of cancer patients are eligible for ICI treatment, and substantial numbers of patients experience durable responses $(5,6)$. Outcomes can be improved by combination of PD-1/PD-L1 with CTLA-4 blockade (7). As of December 2020, seven ICIs had FDA approval (Table 1). The increase in ICI trials from 2250

Conflict of interest: JM has served on scientific advisory boards for Bristol Myers Squibb, Takeda, Deciphera, AstraZeneca, Nektar, Audentes Therapeutics, TripleGene, Boston Biomedical, ImmunoCore, Janssen, Myovant, Cytokinetics, and Amgen. AHS is on the scientific advisory boards for Surface Oncology, SQZ Biotech, Elstar Therapeutics, Elpiscience, Selecta, and Monopteros, consults for Novartis, and has research funding from Merck, Novartis, Roche, Ipsen, and Quark Ventures. AHS has patents/ pending royalties from Roche and Novartis on intellectual property on the PD-1 pathway (patent 7,432,059 with royalties paid from Roche, Merck, Bristol-Myers-Squibb, EMD-Serono, Boehringer-Ingelheim, AstraZeneca, Leica, Mayo Clinic, Dako, and Novartis; patent 7,722,868 with royalties paid from Roche, Merck, Bristol-Myers-Squibb, EMD-Serono, Boehringer-Ingelheim, AstraZeneca, Leica, Mayo Clinic, Dako, and Novartis; patents 8,652,465 and 9,457,080 licensed to Roche; patents 9,683,048, $9,815,898,9,845,356,10,202,454$. and $10,457,733$ licensed to Novartis; and patents $9,580,684,9,988,452$, and $10,370,446$ issued to none). LG has received research funding from Lytix and Phosplatin, as well as consulting/advisory honoraria from Boehringer Ingelheim, AstraZeneca, OmniSEQ, The Longevity Labs, Inzen, and the Luke Heller TECPR2 Foundation. RNK is cofounder and president of ASPIDA Therapeutics Inc. RNK has a patent (PCT/ US2018/021644) for small-molecule BAX inhibitors.

Copyright: ( 2021, American Society for Clinical Investigation.

Reference information: J Clin Invest. 2021;131(5):e145186.

https://doi.org/10.1172/JCI145186. (in 2018) to 3428 (in 2019) reflects the prominence that ICIs have assumed in cancer treatment $(8,9)$.

Given that ICIs act to unleash $\mathrm{T}$ cell responses, it is not surprising that these agents can cause immune-related adverse events (irAEs), some of which can be severe or even fatal (10, 11). While ICI-associated toxicities can affect any organ, myocarditis has emerged as an infrequent, but often lethal, complication $(12,13)$. Other cardiovascular toxicities include pericarditis, vasculitis, arrhythmias (14), and possibly atherosclerosis (15). Cardiovascular irAEs appear to manifest sporadically, but this perception may reflect a poor understanding of predisposing factors and failure to recognize mild presentations. Further, while immune checkpoints are critical in maintaining homeostasis between the immune and cardiovascular systems (16), the precise mechanisms by which ICIs cause cardiotoxicity remain undefined. Here, we provide an overview of ICI-associated myocarditis in the context of immune checkpoint biology. We discuss how immune checkpoints have been successfully manipulated to treat cancer, their roles in protecting the heart from the immune system, and potential mechanisms by which immune checkpoint inhibition may lead to cardiac damage.

\section{Maturation and activation of T cells}

The adaptive immune system generates millions of $\mathrm{T}$ cell clones, each defined by a unique $\mathrm{T}$ cell receptor (TCR) with binding specificity for a particular antigenic ligand. While the T cell lineage arises from a common lymphoid progenitor in the bone marrow, the generation of TCR diversity takes place in the thymus. Through positive and negative selection in response to thymic peptides, $\mathrm{T}$ cell clones emerge that (a) display either CD4 or CD8 - but not 
Table 1. FDA-approved immune checkpoint inhibitors

$\begin{array}{lcc}\text { Drug } & \text { Target } & \text { Approval } \\ \text { Ipilimumab } & \text { CTLA-4 } & 2011 \\ \text { Nivolumab } & \text { PD-1 } & 2014 \\ \text { Pembrolizumab } & \text { PD-1 } & 2014 \\ \text { Atezolizumab } & \text { PD-L1 } & 2016 \\ \text { Durvalumab } & \text { PD-L1 } & 2017 \\ \text { Avelumab } & \text { PD-L1 } & 2017 \\ \text { Cemiplimab } & \text { PD-1 } & 2019\end{array}$

both - on their cell surface; and (b) have the potential to recognize disease-related antigens but, generally, not self-antigens (17, 18). Following further activation events, some clones provide the effector cells $-\mathrm{CD}^{+} \mathrm{T}$ helper (Th) lymphocytes and $\mathrm{CD} 8^{+}$cytotoxic $\mathrm{T}$ lymphocytes (CTLs) - that carry out $\mathrm{T}$ cell-mediated immunity $(19,20)$.

$\mathrm{T}$ cells that emerge from the thymus are mature but naive, meaning that they have not yet encountered their specific antigen. Priming is the first step in T cell activation and takes place in secondary lymphoid organs, such as lymph nodes, spleen, and mucosa-associated lymphoid tissues (ref. 21 and Figure 1). Priming requires both (a) antigen binding to TCR and (b) costimulatory signals. The TCR ligand consists of peptides derived from an antigen in complex with MHC molecules that are displayed on the cell surface (21), particularly on specialized antigenpresenting cells (APCs). However, most disease-related antigens originate in cells of peripheral tissues, whereas $\mathrm{T}$ cell priming takes place in secondary lymphoid organs. Accordingly, APCs, mainly dendritic cells, play a critical role in bringing antigens to priming sites (21). The antigen is processed into peptides by proteasomes or lysosomes that are displayed on the cell surface in the context of class I or class II MHC, respectively (22). Peptides displayed by class I MHC bind to specific TCRs on $\mathrm{CD}^{+} \mathrm{T}$ cells, while those displayed in the context of class II MHC bind to TCRs on $\mathrm{CD}^{+}$cells $(23,24)$.

In addition to TCR engagement, $\mathrm{T}$ cell priming requires additional signals, including binding of costimulatory ligands that reside on the surface of APCs to their receptors on T cells (25). The most intensively studied costimulatory ligands on APCs are CD80 (B7-1) and CD86 (B7-2), both of which bind CD28 on T cells (refs. 26-28 and Figure 1). While CD28 is constitutively present on most mature T cells, CD80 and CD86 expression on APCs is upregulated in response to innate immune danger signals such as microbe-associated and damage-associated molecular patterns emanating from infected or diseased cells, respectively $(29,30)$.

Subsequent steps in the differentiation and activation of $\mathrm{T}$ cell effector functions take place when primed cells circulate to peripheral tissues and re-encounter their specific antigen on the surface of diseased parenchymal cells or on APCs resident in these tissues (Figure 1). In contrast to priming, these later activation steps require only TCR engagement by antigen/MHC, although they are amplified by other costimulatory signals (30). The major effector function of $\mathrm{CD}^{+}$Th cells is to provide such signals by secreting cytokines. Cytokines promote further $\mathrm{T}$ cell expansion and differentiation into Th subtypes, whose functions include the recruitment and activation of various leukocyte populations. While $\mathrm{CD}^{+}$CTLs also secrete cytokines, their major function is to kill target cells (31).

\section{Tolerance to self and immune checkpoints}

The major goal of $\mathrm{T}$ cell-mediated immunity is to recognize microbial and perhaps cancer-related antigens and to kill the microbes or cancer cells by various effector mechanisms. For normal life to exist, however, the immune system must tolerate certain antigens (32). For example, immune tolerance is needed for antigens derived from normal cellular proteins (self-antigens), paternal antigens in the fetus, and some foreign antigens (e.g., commensal bacteria at barrier surfaces and nontoxic environmental chemicals; ref. 33). Accordingly, multiple mechanisms of tolerance have emerged.

One mechanism of central (thymic) tolerance is negative selection against autoreactive $\mathrm{T}$ cell clones (17). Immature thymic $\mathrm{T}$ cells are presented with peptides derived from self-antigens delivered from the circulation by APCs or through the transcription within medullary thymic epithelial cells of multiple genes that are otherwise expressed only in specific tissues. T cells bearing TCRs that do not interact with these antigens fail to receive survival signals and are deleted by apoptosis (negative selection; ref. 34 ). $\mathrm{T}$ cells that bind these antigens with very high affinity also undergo apoptosis (also negative selection). Only T cells with TCRs that bind weakly to these antigens survive (positive selection) $(17,35)$. Mechanistically, it remains unclear why strong antigen-TCR interactions promote apoptosis in immature $\mathrm{T}$ cells in the thymus but activate mature $\mathrm{T}$ cells in peripheral tissues.

Some self-antigens are unrepresented among thymic peptides, and central tolerance is not completely efficient. Accordingly, additional mechanisms of tolerance are needed. One mechanism of peripheral tolerance is provided by decreased costimulatory signals during T cell priming (36). TCR engagement in the absence of adequate costimulation can result in tolerance through anergy (a state of $\mathrm{T}$ cell functional unresponsiveness mediated by cell-intrinsic mechanisms), apoptosis, or inhibition of effector $\mathrm{T}$ cells by a distinct $\mathrm{T}$ cell subset termed "regulatory T cells" (Tregs) (refs. 37, 38; and Figure 1A). One situation that may produce inadequate costimulation is when danger signals are not sufficiently robust to upregulate CD80 and CD86 on APCs. This may be the case in some cancer contexts in contrast to infection or tissue damage.

A second important mechanism of peripheral tolerance is provided by coinhibitory receptors on the surface of $\mathrm{T}$ cells collectively referred to as immune checkpoints (ref. 36 and Figure 1). One of the best-characterized coinhibitory receptors is cytotoxic $\mathrm{T}$ lymphocyte-associated protein 4 (CTLA-4; CD152; refs. 36, 39). CTLA-4, which is homologous to CD28, similarly binds CD80 and CD86, but with much higher affinity than CD28 (40). Hence, depending on the relative abundances of CTLA- 4 and CD28 and whether CD80 and CD86 are present at limiting levels, CTLA-4 can outcompete CD28 for CD80 and CD86 (41). While CD28 is expressed on most mature T cells, CTLA-4 levels are transiently upregulated on mature $\mathrm{CD} 4^{+}$and $\mathrm{CD} 8^{+}$cells during activation $(42,43)$. Increased CTLA-4 levels coupled with a higher affinity 

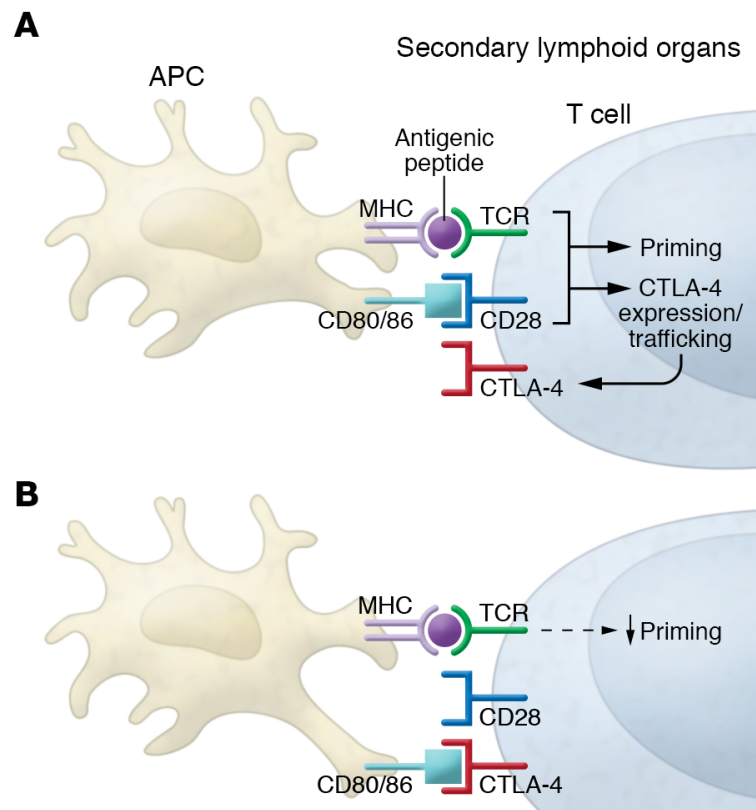

Peripheral tissues
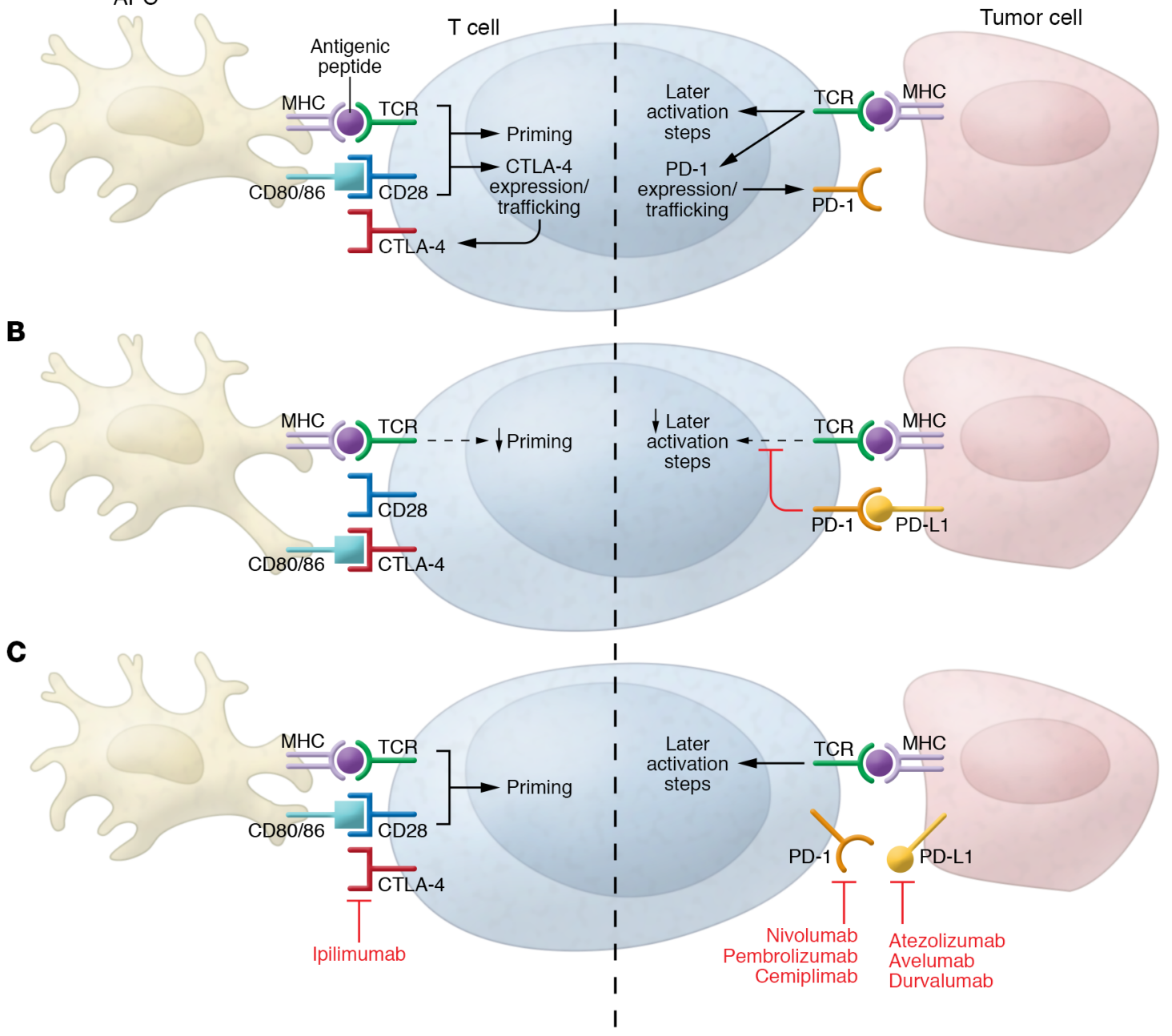

Figure 1. Immune checkpoints in T cell priming and activation. (A) Mature, but naive, T cells are primed in secondary lymphoid organs. The priming process requires engagement of the TCR by its specific antigenic ligand, which consists of antigen-derived peptides displayed on the surface of antigenpresenting cells (APCs) in the context of MHC molecules; and costimulatory signals provided, in part, by binding of CD80/86 on the surface of APCs to CD28, which is constitutively present on T cells. Full effector functions are acquired in peripheral tissues when the TCR re-encounters its specific antigenic ligand. Priming and later activation steps also result in induction of CTLA-4 and PD-1, respectively, which are coinhibitory receptors that are expressed on the surface of T cells and function as immune checkpoints. (B) CTLA-4 outcompetes CD28 for binding to CD80/86, thereby attenuating CD28-mediated costimulation, reflecting the stronger affinity of CD80/86 for CTLA-4 as compared with CD28 and the upregulation of CTLA-4 during priming. Following binding by its ligands, PD-L1 and PD-L2 (not shown), PD-1 suppresses T cell activation through cell-intrinsic mechanisms that disrupt signaling downstream of TCR (see main text). Genetic alterations or interferon stimulation in cancer cells can induce PD-L1, providing a mechanism for these cells to evade killing by the immune system. IFN- $\gamma$-induced expression of PD-L1 on cardiac endothelial cells also provides a means for the heart to protect itself against T cells. Not shown is the role of Tregs (see main text). (C) Approved ICls are monoclonal antibodies that bind CTLA-4, PD-1, or PD-L1, thereby disrupting interactions between CD80/86 and CTLA-4 and between PD-1 and PD-L1, respectively.

for CD80 and CD86 result in CTLA-4 stealing these costimulatory ligands from CD28 (44-46). While CTLA-4 may also possess some cell-intrinsic signaling functions, sequestration of CD80 and CD86 away from CD28 is thought to be the major mechanism by which CTLA-4 inhibits $\mathrm{T}$ cell activation $(26,36)$.

In addition to affecting $\mathrm{T}$ cell priming, CTLA-4 also inhibits effector $\mathrm{T}$ cells in peripheral tissues through indirect effects involving Tregs (47). Tregs, most of which are $\mathrm{CD} 4{ }^{+} \mathrm{CD} 25^{+}$and express the FOXP3 transcription factor, arise primarily in the thymus but also in peripheral tissues $(48,49)$. Tregs act to inhibit other immune cells ( $\mathrm{T}$ cells, B cells, and NK cells) through a variety of mechanisms, including the secretion of inhibitory cytokines (e.g., IL-10) and competition for factors that would otherwise stimulate effector T cells (e.g., IL-2, CD80/CD86; ref. 48). Importantly, Tregs constitutively express CTLA-4, which is critical for their inhibitory function $(3,47)$. Accordingly, CTLA-4 inhibits $\mathrm{T}$ cell activation directly during priming in secondary lymphoid organs and indirectly through Tregs at later steps in activation in peripheral tissues.

The importance of CTLA-4 in restraining T cells in vivo is underscored by knockout mice $(50,51)$. Germline deletion of Ctla4 results in death early in life due to lymphoproliferation and multiorgan, $\mathrm{T}$ cell-mediated inflammatory disease $(50,51)$. A similar, albeit delayed, lethal diffuse inflammatory disease is 
also seen in mice lacking Ctla4 specifically in Tregs (47). Further, human genetic variants of CTLA4 are associated with autoimmune disorders, consistent with the critical role of CTLA-4 in tolerance $(52,53)$. These patients have heterozygous CTLA4 mutations associated with Treg dysregulation, effector $\mathrm{T}$ cell hyperactivation, and lymphocytic tissue infiltration.

A second coinhibitory TCR important in peripheral tolerance is programmed cell death protein-1 (PDCD1; PD-1; Figure 1). PD-1 has two major ligands, PD-L1 (CD274; B7-H1) and PD-L2 (CD273; B7-DC; refs. 54, 55). PD-L1 is expressed broadly, including on APCs, macrophages, endothelial cells, cells in peripheral tissues, and cancer cells, and is induced by IFN- $\alpha$, $-\beta$, and $-\gamma(54,56)$. In contrast, PD-L2 expression is restricted to APCs (including dendritic cells, macrophages, and B cells), specific subsets of Th cells, and airway epithelia $(54,57)$. Antigen engagement of TCR transiently increases PD-1 on T cells. Following ligand binding to $\mathrm{PD}-1$, specific tyrosine residues on its cytoplasmic tail undergo phosphorylation. This phosphorylation results in recruitment of a protein tyrosine phosphatase that antagonizes signaling events important for $\mathrm{T}$ cell stimulation downstream of TCR and CD28 (58). Thus, in contrast to CTLA-4, which inhibits mainly by stealing CD80 and CD86 ligands on APCs away from CD28, PD-1 inhibits the activation of effector $\mathrm{T}$ cells through cell-intrinsic signaling. Germline deletion of $P d c d 1$ in mice can lead to autoimmune phenotypes, albeit milder than those resulting from Ctla4 deletion and dependent on genetic background (discussed below; refs. $59,60)$. In addition to effector T cells, PD-1 is also expressed on Tregs (61). However, in contrast to the role of CTLA-4 in promoting the immunosuppressive functions of Tregs, PD-1 restrains immunosuppression by these cells (62).

Other costimulatory and coinhibitory receptors also regulate $\mathrm{T}$ cell responses. Costimulatory receptors include inducible T cell costimulator (ICOS; CD278), 4-1BB (CD137), and OX40 (CD134); and coinhibitory receptors include lymphocyte activation gene-3 (LAG-3; refs. 63, 64), T cell immunoglobulin and mucin domain-containing-3 (TIM-3), and T cell immunoreceptor with immunoglobulin and immunoreceptor tyrosine-based inhibitory motif domains (TIGIT; refs. 65-67). While the molecular mechanisms by which these receptors act are diverse and incompletely understood, their existence underscores the complexity of $\mathrm{T}$ cell regulation. These receptors are also targets for anticancer immunotherapies under development, including agonistic antibodies against costimulatory receptors and blocking antibodies against coinhibitory receptors (68). The ability to therapeutically modulate these additional targets will likely be important because cancers often evolve to hijack $\mathrm{T}$ cell regulatory mechanisms to their own advantage (69).

\section{Immune checkpoint inhibitors as cancer therapy}

Over the last decade, CTLA-4, PD-1, and PD-L1 have been successfully targeted by anticancer therapeutics commonly referred to as immune checkpoint inhibitors (ICIs) or blockers (ref. 1 and Figure $1 \mathrm{C}$ ). The potential of anti-CTLA-4 therapy was initially recognized by its robust effects in immunocompetent, syngeneic mouse tumor models (70). Similarly, early work showed that enforced expression of PD-L1 on tumor cells renders them less susceptible to lysis by CD8 ${ }^{+} \mathrm{T}$ cells (71-73). Conversely, PD-1 or PD-L1 blockade potentiates antitumor immunity $(72,74)$. Intensive investigation focusing on the development of antibodies against CTLA-4, PD-1, and PD-L1 for cancer therapy followed. Binding of anti-CTLA-4 and anti-PD-1 to CTLA-4 and PD-1, respectively, precludes CTLA-4 and PD-1 from interacting with their individual ligands (75). Similarly, anti-PD-L1 prevents PD-L1 from binding PD-1.

Initial clinical testing of ipilimumab, an anti-CTLA-4 antibody, demonstrated durable responses in some melanoma patients with considerable increases in survival (76). PD-1 and PD-L1 blockers mediated similar responses in patients with advanced and otherwise untreatable melanoma, non-small cell lung carcinoma, and urothelial tumors $(77,78)$. However, both CTLA-4 blockade and PD-1/PD-L1 blockade were associated with high-grade irAEs, including colitis, dermatitis, thyroiditis, pneumonitis, and hepatitis (79-81). These toxicities presumably arose from ICI-induced failure of self-tolerance $(10,82)$. The complementary immunosuppressive effects of CTLA-4 and PD-1 supported the implementation of combinatorial therapeutic targeting. The combination of ipilimumab and nivolumab (antiPD-1) was associated with increased response rate in patients with melanoma and renal carcinoma $(7,83)$, although at the cost of more frequent high-grade irAEs (81).

These early results have led to an explosion of clinical trials testing ICIs for multiple cancers and using diverse strategies $(2,84)$. Combinatorial ICI treatments extend beyond CTLA-4 and PD-1/PD-L1 pathways. Antibodies targeting the coinhibitory receptors LAG-3, TIM-3, and TIGIT have been combined with anti-PD-1 therapy $(64,68)$. In addition, in many cancer types, ICIs are combined with traditional chemotherapy, radiation, angiogenesis inhibitors, and targeted anticancer agents (2, 84-89). Finally, ICI-based therapies have become first-line therapeutics in both adjuvant (given after the primary treatment to lower recurrence risk) and neoadjuvant (initial treatment as first step prior to the main treatment, usually surgery) settings, in addition to previous indications for advanced, disseminated, and treatment-resistant disease $(90,91)$.

Increasing recognition has developed of the potential severity and lethality of ICI-associated toxicities $(11,92)$. Fatalities appear to occur randomly and often early following initiation of therapy (11). Interestingly, the spectrum of fatal organ involvement differs between anti-CTLA-4 and anti-PD-1/PD-L1 therapies, with colitis most often reported in the former and pneumonitis, hepatitis, and neurotoxicity in the latter (93-95). Deaths from combination treatment (e.g., anti-PD-1/CTLA-4) most frequently result from colitis or myocarditis (11). Among irAEs, myocarditis has the highest fatality rate, $40 \%$ to $50 \%$ depending on the study $(11,14,96)$. These observations underscore the need for strategies that mitigate ICI-associated toxicities without undermining anticancer efficacy.

\section{Adverse cardiac events resulting from ICls}

In 2016, Johnson et al. reported two cases of fulminant myocarditis following treatment with ICIs, described the incidence of myocarditis in a retrospective clinical trial population, and 
A

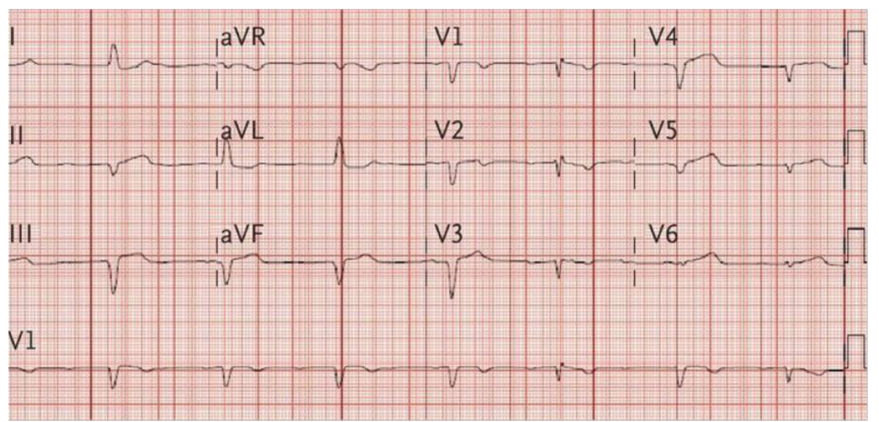

C

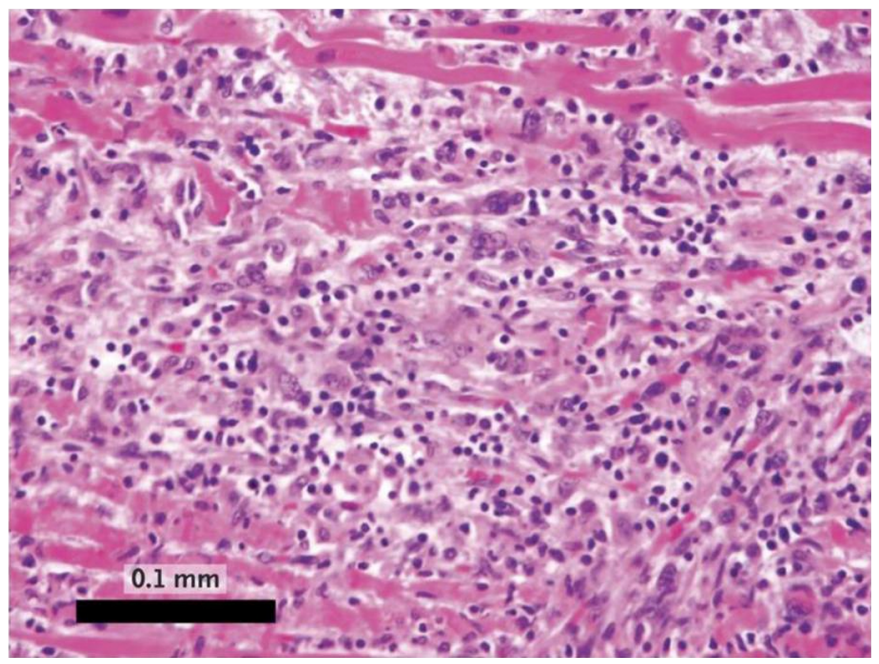

B

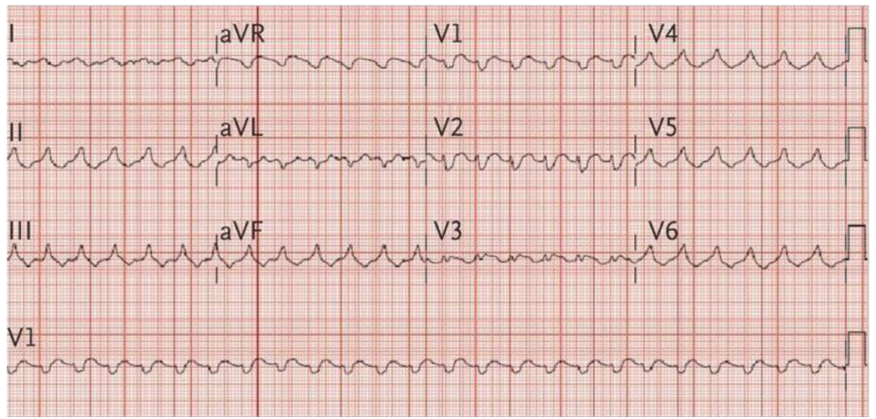

D

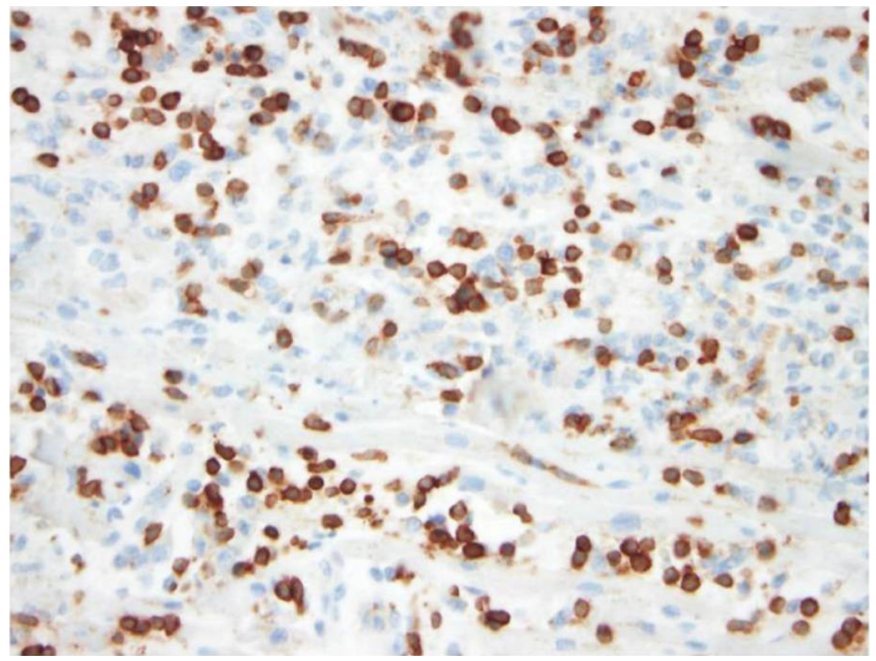

Figure 2. Example of ICl-associated myocarditis. A 65-year-old woman with metastatic melanoma treated with ipilimumab ( $3 \mathrm{mg} / \mathrm{kg}$ i.v.) and nivolumab ( $3 \mathrm{mg} / \mathrm{kg}$ i.v.) developed atypical chest pain, dyspnea, and fatigue 12 days later, accompanied by (A) ECG showing sinus rhythm with first-degree atrioventricular block, which progressed to complete heart block. (B) Several hours later, ECG showed ventricular tachycardia, which degenerated to ventricular fibrillation and cardiac arrest. The patient could not be resuscitated. (C) At autopsy, cardiac tissue stained with hematoxylin and eosin showed myocyte degeneration accompanied by a mononuclear cell infiltrate, immunostaining of which showed prominence of CD68, a macrophage marker (not shown). (D) Also seen was marked infiltration of T cells, as shown by immunostaining for CD3. This infiltrate included approximately equal proportions of CD4+ and CD8 ${ }^{+} T$ cells (not shown). In addition, immunostaining for CD2O, a B cell marker, and IgG was not detected (not shown). Scale bar: 0.1 mm. aVR, augmented vector right; aVL, augmented vector left; aVF, augmented vector foot. Reproduced with permission from the New England Journal of Medicine (13).

defined basic clinical and pathophysiological characteristics of the syndrome (13). Multiple case series have since been added $(14,96-98)$. Additionally, interrogation of individual case safety reports from publicly available databases indicates an increased incidence of myocarditis in patients receiving ICIs (96).

The clinical presentation of ICI-associated myocarditis is variable. Fulminant cases are characterized by early onset following treatment, arrhythmias/conduction disturbances, concomitant skeletal myositis and myasthenia gravis, and high mortality (refs. 14, 96; and Figure 2). While heart damage results in cardiac biomarkers of injury (e.g., increased serum troponin concentrations), surprisingly, nearly $50 \%$ of patients exhibit no evidence of systolic dysfunction (97). On the other hand, cardiac arrhythmias are common, including atrial fibrillation, ventricular arrhythmias, conduction abnormalities, and sudden death $(97,98)$. ICIs can also lead to other cardiovascular irAEs, including pericarditis and vasculitis $(12,14)$. In addition, case reports suggest that other forms of cardiomyopathy associate with ICI treatment, including Takotsubo syndrome, which may resemble myocarditis (99). The concomitant presence of skeletal myositis and myasthenia gravis following ICI administration should raise suspicion for myocarditis (100, 101). In contrast to fulminant disease, "smoldering" cases of ICI-associated myocarditis have also been reported (102). The long-term consequences of such cases are unclear. Given the growing number of cancer survivors, a future challenge for oncologists and cardiologists will be to understand long-term cardiovascular effects of ICIs (103).

Diagnosing myocarditis can be challenging, especially in patients treated with ICIs. As a result, a multipronged approach has been proposed to rule out other causes for symptomatology (such as acute coronary syndrome) and include a combination of biomarker tests, cardiac imaging, and biopsy (104). Traditionally, endomyocardial biopsy has been the gold standard for diagnosing myocarditis $(105,106)$, and it should be performed in most cases. The histopathological features of ICI-associated myocarditis involve myocardial infiltration of $\mathrm{T}$ lymphocytes (both $\mathrm{CD} 4^{+}$and $\mathrm{CD} 8^{+}$) and macrophages, and myocyte death; B lymphocytes have 
not been noted (ref. 13 and Figure 2). These features are consistent with the Dallas criteria for the diagnosis of myocarditis (107). Cardiac MRI may further clarify diagnosis $(105,108)$.

The incidence of ICI-associated myocarditis remains uncertain. Early ICI-based cancer trials did not prospectively screen for myocarditis (109). In addition, because diagnosing myocarditis can be difficult, cases in these trials could easily have been missed. Reports suggest that the incidence of ICI-associated myocarditis is $0.27 \%$ to $1.14 \%(13,97)$. However, the true incidence may be higher, as initial reports identified only severe cases (102). Also of unclear significance are cases in which there is an increase in serum troponin concentration following ICI treatment (called "troponitis"), indicating some cardiac damage, but without obvious cardiovascular symptoms. One single-center study reported abnormal serum troponin I concentrations in 13 of 76 patients, with unclear clinical implications (110). Therefore, identification and long-term prognostication of smoldering forms of myocarditis are needed.

Few risk factors for ICI-associated myocarditis have been identified. Combination of anti-CTLA-4 and anti-PD-1 appears to be the strongest risk factor, with pharmacovigilance data suggesting that the combination of ipilimumab and nivolumab is accompanied by a 4.74 -fold risk of myocarditis compared with nivolumab alone (13). It is conceivable that cardiac-, immune-, and cancerrelated factors impact risk for ICI-associated myocarditis (111). Additionally, genetic variation, including germline or somatic (e.g., in the tumor), may contribute. Studies involving large numbers of patients are required to dissect predisposing factors for myocarditis and other ICI-associated cardiac pathologies.

Identifying patient populations at risk for myocarditis has clear clinical implications. First, while ICIs were approved for advanced malignancies, they are now being tested in front-line settings where the net benefit of therapy may be ill defined. Second, the use of ICIs in combination with other cardiotoxic drugs likely elevates risk (112). Given these potentially complicated scenarios, a more fundamental understanding of pathogenesis is essential.

Currently, no consensus exists regarding screening, surveillance, prevention, or treatment strategies for ICI-associated myocarditis. Baseline electrocardiogram and troponin levels are probably indicated in all patients $(12,111,113,114)$, and high-risk patients should probably undergo surveillance with serum troponin concentrations after the initiation of treatment. However, the fact that increased serum troponin concentrations are difficult to interpret in asymptomatic patients underscores the need for improved predictive biomarkers. Prospective, multi-institutional efforts are needed to identify patients at high risk for ICI-associated myocarditis as well as the best means to surveil them.

Treatments for myocarditis have been largely extrapolated from therapies for noncardiac ICI-associated toxicities, including cessation of ICIs, supportive management, and glucocorticoids (115). Generally, irAEs are treated with prednisone ranging from 0.5 to $2.0 \mathrm{mg} / \mathrm{kg}$ (or equivalent), followed by a 4 - to 6-week taper upon symptom improvement $(115,116)$. In the case of myocarditis, higher steroid doses (methylprednisolone, $1 \mathrm{~g}$ ) have been advocated (117). Nevertheless, data from public databases indicate that mortality remains substantial. Individual case reports demonstrate successful treatment with other therapies including mycophenolate mofetil, tacrolimus, alemtuzumab, or abatacept (118-122). While these medications are all considered immunosuppressive therapies, their specific mechanisms of action differ (123). For example, abatacept is a soluble protein consisting of the human CTLA-4 extracellular domain fused to the Fc portion of IgG and functions as a sink for CD80 and CD86 on APCs to limit costimulatory signals $(28,124)$. Preclinical data suggest efficacy of abatacept in the treatment of ICI-associated myocarditis (125), and prospective clinical trials are needed to compare its efficacy with those of other immunosuppressive therapies. Conversely, while some have advocated TNF- $\alpha$ antagonists (like infliximab) for irAEs, concerns have been raised about the use of these agents in patients with heart failure (126).

Although the focus of this Review is ICIs, we note that these agents represent only one class of immuno-oncology therapies, which also include cytokines, vaccines, oncolytic viruses, bispecific molecules, and cellular therapies (127). One would anticipate the possibility of irAEs resulting from any of these. An example is provided by the cellular therapy referred to as chimeric antigen receptor $\mathrm{T}$ (CAR-T) cells, $\mathrm{T}$ cells engineered to express a receptor that targets a specific antigen of interest (e.g., cancer antigen) independent of its presentation on MHC molecules to allow more effective cancer cell killing (128). Thus far, CAR-T cell therapy has shown the greatest benefit in hematopoietic malignancies such as B cell acute lymphoblastic leukemia (B-ALL), in which CD19, an antigen on malignant and differentiated B cells, has been targeted. Of B-ALL patients who have failed previous therapy, $70 \%-90 \%$ experience complete responses to CAR-T cell therapy, whereas traditionally fewer than $10 \%$ of patients survive beyond 5 years (129-132). However, as with ICIs, CAR-T cell therapies have resulted in cardiac complications. In one trial, CAR-T cells against the cell surface protein melanoma-associated antigen-3 (MAGE-A3) resulted in fatal cardiogenic shock in two patients (133). Histological studies demonstrated myocardial damage with considerable $\mathrm{T}$ cell infiltration. MAGE-A3 expression was undetected in cardiac tissue. Rather, CAR-T cells targeted the sarcomeric protein titin $(133,134)$. In addition to raising questions as to why and how titin was recognized, this example illustrates the potential of cardiotoxicity from any immune cancer therapy. It will be incumbent on clinicians to have a low threshold of suspicion as each new agent becomes available.

\section{Immune-cardiac interrelationships}

Immune responses, which typically result in inflammation and tissue damage, are particularly dangerous in the heart. Indeed, autoimmune and viral myocarditis can promote fatal arrhythmias and severe contractile dysfunction/heart failure $(107,135)$. The heart is especially vulnerable to immune-mediated damage because its dense vascularity provides access to immune cells and antibodies, its anatomy is nonredundant, and even small foci of damage can provide a substrate for arrhythmias.

Mechanisms exist that dampen baseline and stimulated immune function in the heart as in other immune-privileged organs (136). First, while the myocardium contains macrophages and dendritic cells, which are thought to play roles in tissue repair (137), relatively few $\mathrm{T}$ cells are present at least under 
basal conditions. Second, both central and peripheral tolerance mechanisms limit $\mathrm{T}$ cells directed against myocardial antigens. Third, T cell-mediated injury to the heart is reduced by negativefeedback loops that operate in the myocardium. These feedback loops include the secretion of the cytokine IFN- $\gamma$ from Th1 cells and CTLs, resulting in PD-L1 upregulation on cardiac endothelial cells; PD-L1 then suppresses effector T cells (138). IFN- $\gamma$ also induces differentiation of a monocyte-derived dendritic cell, resulting in abundant nitric oxide production, which blocks further differentiation and expansion of Th1 and Th17, Th subsets involved in myocarditis (139).

\section{Mechanisms of ICl-associated myocarditis}

The mechanistic basis of ICI-associated myocarditis is not understood. While existing data support $\mathrm{T}$ cell-mediated immunity as a critical component in pathogenesis, many questions remain: (a) What are the inciting cardiac antigens? (b) Why do these self-antigens elicit an immune response? (c) What additional roles are played by innate immune and B cells? (d) What cardiac cell types are involved? (e) What aspects of cardiac cellular dysfunction are manifest? (f) Is cell death a critical component of pathogenesis, and, if so, which cell death programs are involved? (g) Does the predominance of arrhythmias primarily reflect damage to the conduction system or generalized inflammation? (h) Is inflammation the major myocardial phenotype, or does dilated cardiomyopathy ensue as in some patients with viral and autoimmune myocarditis?

The importance of $\mathrm{T}$ cells, and immune checkpoints in particular, is supported by genetic loss-of-function studies in mice. As discussed above, germline or Treg-specific deletion of Ctla4 results in lymphoproliferation, diffuse tissue inflammation including myocarditis, and premature mortality $(50,51)$. In contrast, disruption of the PD-L/PD-1 axis in mice results in variable cardiac phenotypes that appear to be dependent on genetic background and possibly environmental factors. Specifically, germline deletion of $P d c d 1$ (encoding PD-1) on a C57BL/6 background does not elicit a cardiac phenotype, although mild signs of arthritis and glomerulonephritis emerge in some older mice (59). However, one germline $P d c d 1$ knockout in mice on a BALB/c background resulted in marked cardiac dilation and systolic dysfunction (i.e., dilated cardiomyopathy) (140). While scattered myocyte degeneration and fibrosis were present, myocarditis was notably absent. IgG antibodies against cardiac troponin I were found in the circulation with IgG deposits on the surface of cardiomyocytes, where they were thought to enhance L-type $\mathrm{Ca}^{2+}$ channel currents (141). Moreover, dilated cardiomyopathy could be phenocopied by injection of monoclonal anti-cardiac troponin I antibodies. However, an independently generated germline $P d c d 1$ knockout, also on a BALB/c background, demonstrated no baseline cardiac abnormalities, including the absence of dilated cardiomyopathy and myocarditis, despite developing cardiac inflammation when challenged with an immune stimulus (142). Consistent with this second $P d c d 1$ knockout, combined germline deletion of the genes encoding PD-L1 and PD-L2 on the C57BL/6 background did not result in any cardiac phenotype or mortality out to 8 months of age (143). The reason for the discrepant phenotypes of the two $P d c d 1$ knockouts on the same background is unknown, although they could reflect differences in targeting strategies or environmental factors.

Although not yet explored in the context of ICI-associated myocarditis, environment unsurprisingly impacts disease pathogenesis in autoimmune myocarditis. A recent example is the role of peptide mimetics of $\alpha$-myosin heavy chain from commensal Bacteroides species, which activate Th17 cells in the gut to promote the transition from myocarditis to lethal cardiomyopathy (144). This mechanism was demonstrated in mice with $\mathrm{CD}^{+}$ cells engineered to express a TCR against $\alpha$-myosin heavy chain, but clinical correlates were also observed in humans with myocarditis $(144,145)$. Antibiotic treatment blocked the transition to heart failure in the mouse model, suggesting that these findings are translationally important.

Although not explaining the discrepant basal cardiac phenotypes of the two Pdcd1-knockout mice on the same (BALB/c) background discussed above, genetic factors exert a major impact on manifestations resulting from disruption of the PD-1 axis. For example, the PD-1 and PD-L1/PD-L2 knockout studies described above were performed on genetic backgrounds that are relatively autoimmune resistant (e.g., BALB/c or $\mathrm{C} 57 \mathrm{BL} / 6$ ). In contrast, studies performed on autoimmune-prone backgrounds (e.g., $\mathrm{MRL}^{-\mathrm{Mpj}^{+/+}}{ }^{+}$refs. 146,147$)$ or in combination with deficiencies in other immune checkpoints (e.g., LAG-3; ref. 148) revealed that loss of PD-1 or PD-L1 can promote myocarditis. These data suggest that redundant mechanisms exist in some genetic backgrounds that compensate for PD-1 loss at least in the basal state. Under immune-stimulated conditions, however, disruption of PD-L1/PD-1 signaling can exacerbate myocarditis eve $109 \mathrm{n}$ on non-autoimmune-prone genetic backgrounds $(138,142)$.

Another recently described mouse myocarditis model also illustrates this point. These mice have monoallelic deletion of Ctla4 superimposed on homozygous loss of $P d c d 1$ (125), which is of translational interest because they provide a preclinical model of combined anti-CTLA-4/anti-PD-1 therapy, an important risk factor for ICI-associated myocarditis (14). Approximately half of these mice die by 3 months of age and manifest severe electrocardiographic abnormalities accompanied by myocardial infiltration by $\mathrm{T}$ cells and macrophages, closely recapitulating the clinical and pathological hallmarks of ICI-associated myocarditis observed in patients. This model suggests that Ctla4 and $P d c d 1$ functionally interact in a gene dosage-dependent manner, providing a mechanism by which myocarditis arises with increased frequency in the setting of combination ICI therapy. In addition, intervention with CTLA-4-Ig (abatacept) ameliorates disease progression in this model, consistent with clinical case reports that abatacept attenuates fulminant ICI-associated myocarditis (121).

The next leading questions unfurl: Which (presumably self) antigens elicit $\mathrm{T}$ cell responses during ICI-associated myocarditis, and why have these antigens escaped tolerance? A variety of cardiac proteins have been associated with autoimmune myocarditis; some are thought to be mediated by antibody-related and others by $\mathrm{T}$ cell-related mechanisms (149). These antigens include cardiac myosin $(145,150-155)$ and $\beta$-adrenergic receptors (156-158). Moreover, in addition to functioning as an antigen, myosin has been shown to function as a damage-associated molecular pattern $(155,159)$. 
Deep sequencing of the CDR3 region (important for antigen binding) of the TCR $\beta$ chain of two melanoma patients who were treated with combined anti-CTLA-4/anti-PD-1 therapy and went on to develop fatal myocarditis and skeletal myositis showed considerable overlap in $\mathrm{T}$ cell clonotypes in cancers, heart, and skeletal muscle (13). The antigen target(s) of these TCRs remain unidentified. However, bulk RNA sequencing of cancer tissue identified high levels of striated muscle transcripts, raising the possibility that antigenic peptides derived from the muscle proteins elicited a $\mathrm{T}$ cell response that secondarily damaged cardiac and skeletal muscle. A second possibility is that a relevant cancer antigen is expressed in cardiac cells. A third possibility is molecular mimicry in which a cancer antigen-directed TCR may recognize a cardiac antigen that bears structural similarity at baseline or following posttranslational modification. Interestingly, molecular mimicry has been demonstrated between microbial antigens and cardiac proteins in rheumatic heart disease and viral myocarditis (153, 160-163) and even between cardiac myosin and cardiac $\beta$-adrenergic receptors in autoimmune myocarditis and dilated cardiomyopathy $(156,158)$. Recognition of structural similarities between antigens can be challenging $(133,134,164)$, and the specificity of TCR binding for a particular antigen can also be impacted by the MHC context (165).

Why would these antigens slip through the central and peripheral mechanisms that promote tolerance to self? While, in most cases, cause-and-effect relationships remain unclear (136), this question has been explored for $\alpha$-myosin heavy chain (166). Transcripts encoding $\alpha$-myosin heavy chain are undetectable in human or mouse medullary thymic epithelial cells. Further, transgenic expression of this gene in the thymic epithelium suffices to suppress myocarditis in a mouse model mediated by $\alpha$-myosin heavy chain-specific $\mathrm{CD} 4^{+} \mathrm{T}$ cells. While these data suggest that absence of central tolerance may contribute to immune recognition of self in this model, they do not explain why mechanisms of peripheral tolerance fail to compensate.

Multiple additional questions pertain to the heart injury itself. Given the observed elevations in serum troponin concentrations, there is little question that cardiomyocytes are injured and undergo cell death in ICI-associated myocarditis. However, the extent to which this injury constitutes a direct attack by the immune system on cardiomyocytes versus damage inflicted indirectly through compromise of other cardiac cell types, such as endothelial cells, remains unclear. Similarly, direct involvement of the conduction system has been observed histologically in ICI-associated myocarditis and likely contributes to the prominence of arrhythmias $(13,98)$. Information is not yet available, however, concerning the relative contributions of direct injury versus reactive fibrosis in producing rhythm disturbances. Finally, an explanation is warranted for the seemingly low incidence of heart failure in ICI-associated myocarditis, which contrasts with some patients with viral and autoimmune myocarditis, in which the inflammatory phase transitions to dilated cardiomyopathy (167). Whether the apparent low incidence of heart failure with ICIs reflects rapid mortality from fulminant myocarditis or mechanistic differences in cardiac signaling in comparison with other forms of myocarditis merits further investigation.

A third set of questions revolve around how the immune system damages the heart. One central question is whether the major outcome is cardiac cell death, cell dysfunction, or both, the answer to which may depend on which cardiac cell types are involved. T cells induce cancer cell death through several mechanisms (168, 169): (a) the engagement of death receptors on cancer cells by death ligands resident in the plasma membrane of CTLs (170); and (b) secretion of perforin-1 and granzyme B from CTLs (171). Perforin creates pores within the cancer cell plasma membrane that allow entry of granzyme B, a serine protease that induces cell death through caspase activation and other mechanisms $(169,170)$. In addition, recent work has implicated additional death programs, such as ferroptosis, in the killing of cancers by CTLs (172). The extent to which these mechanisms are involved in ICI-associated myocarditis is not known. Cytokines have also been suggested to induce cardiac cell dysfunction and death during the postinfection phase of viral myocarditis (173), and similar mechanisms may operate in the context of cell-based immunotherapies (174-177). If loss of viability of cardiac cells proves to be an important component of ICI-associated myocarditis, it will be important to determine the cell death programs and regulatory mechanisms.

\section{Future directions}

The emergence of ICI-associated myocarditis as a clinical entity has raised multiple questions. One is the extent to which this cardiotoxicity will limit deployment of these agents. Given the apparent low frequency of ICI-associated myocarditis, one would not anticipate this possibility were it not for the lethality of this complication. Accordingly, studies are needed to determine the frequency of ICI-associated myocarditis more precisely. Second, work is needed to identify markers predictive of lethality in ICI-associated myocarditis. Third, more extensive clinical data are needed to sharpen diagnostic and therapeutic protocols. For example, is endomyocardial biopsy always needed for diagnosis? What is the relative balance between mitigating cardiac complications and potentially undermining cancer treatment? Perhaps most important because of its likely impact on diagnosis and treatment, an understanding of the pathogenesis of ICI-associated myocarditis at the molecular and cellular levels is needed. Although genetic mouse models have been invaluable, studies involving blood and tissues obtained from patients will be critical for understanding mechanisms.

\section{Acknowledgments}

This work was supported by the NIH (R01HL141466 to JM, R01HL131862 to AHL, P01AI56299 to AHS, P01AI039671 to AHS, R01H130861 to RNK, and R01HL138475 to RNK); the US Department of Defense (C180476P1 to LG and PR191593 to RNK); the American Heart Association (18SRG34280018 to RNK); and Foundation Leducq (RA15CVD04 to RNK). The authors also acknowledge helpful discussions with Pilar Alcaide, Nikolaos G. Frangogiannis, Sally Huber, Kirk U. Knowlton, Bruce M. McManus, and Sumanth Prabhu.

Address correspondence to: Javid Moslehi, Vanderbilt University Medical Center, 2220 Pierce Avenue, Nashville, Tennessee 37232, USA. Phone: 615.343.9436; Email: javid.moslehi@vumc. org. Or to: Richard N. Kitsis, Albert Einstein College of Medicine, 1300 Morris Park Avenue, Bronx, New York 10461, USA. Phone: 718.430.2609; Email: richard.kitsis@einsteinmed.org. 
1. Ribas A, Wolchok JD. Cancer immunotherapy using checkpoint blockade. Science. 2018;359(6382):1350-1355.

2. Galluzzi L, et al. Immunostimulation with chemotherapy in the era of immune checkpoint inhibitors. Nat Rev Clin Oncol. 2020;17(12):725-741.

3. Wei SC, et al. Fundamental mechanisms of immune checkpoint blockade therapy. Cancer Discov. 2018;8(9):1069-1086.

4. Sharma P, Allison JP. Dissecting the mechanisms of immune checkpoint therapy. Nat Rev Immunol. 2020;20(2):75-76.

5. Kalbasi A, Ribas A. Tumour-intrinsic resistance to immune checkpoint blockade. Nat Rev Immunol. 2020;20(1):25-39.

6. Galluzzi L, et al. The hallmarks of successful anticancer immunotherapy. Sci Transl Med. 2018;10(459):eaat7807.

7. Postow MA, et al. Nivolumab and ipilimumab versus ipilimumab in untreated melanoma. N Engl JMed. 2015;372(21):2006-2017.

8. Tang J, et al. Trial watch: The clinical trial landscape for PD1/PDL1 immune checkpoint inhibitors. Nat Rev Drug Discov. 2018;17(12):854-855.

9. Xin $\mathrm{Yu}$ J, et al. Immuno-oncology drug development goes global. Nat Rev Drug Discov. 2019;18(12):899-900.

10. Postow MA, et al. Immune-related adverse events associated with immune checkpoint blockade. N Engl J Med. 2018;378(2):158-168.

11. Wang DY, et al. Fatal toxic effects associated with immune checkpoint inhibitors: a systematic review and meta-analysis. JAMA Oncol. 2018;4(12):1721-1728.

12. $\mathrm{Hu} \mathrm{JR}$, et al. Cardiovascular toxicities associated with immune checkpoint inhibitors. Cardiovasc Res. 2019;115(5):854-868.

13. Johnson DB, et al. Fulminant myocarditis with combination immune checkpoint blockade. NEngl JMed. 2016;375(18):1749-1755.

14. Salem JE, et al. Cardiovascular toxicities associated with immune checkpoint inhibitors: an observational, retrospective, pharmacovigilance study. Lancet Oncol. 2018;19(12):1579-1589.

15. Drobni ZD, et al. Association between immune checkpoint inhibitors with cardiovascular events and atherosclerotic plaque. Circulation. 2020;142(24):2299-2311.

16. Lichtman AH. The heart of the matter: protection of the myocardium from T cells. J Autoimmun 2013;45:90-96

17. Stritesky GL, et al. Selection of self-reactive T cells in the thymus. Annu Rev Immunol. 2012;30:95-114.

18. Klein L, et al. Positive and negative selection of the T cell repertoire: what thymocytes see (and don't see). Nat Rev Immunol. 2014;14(6):377-391.

19. Singer A, et al. Lineage fate and intense debate: myths, models and mechanisms of CD4- versus CD8-lineage choice. Nat Rev Immunol. 2008;8(10):788-801

20. Carpenter AC, Bosselut R. Decision checkpoints in the thymus. Nat Immunol. 2010;11(8):666-673.

21. Grossman Z, Paul WE. Dynamic tuning of lymphocytes: physiological basis, mechanisms, and function. Annu Rev Immunol. 2015;33:677-713.

22. Neefjes J, et al. Towards a systems understanding of MHC class I and MHC class II antigen presentation. Nat Rev Immunol. 2011;11(12):823-836.

23. Gay D, et al. Functional interaction between human T-cell protein CD4 and the major histocompatibility complex HLA-DR antigen. Nature. 1987;328(6131):626-629.

24. Marusic-Galesic S, et al. Development of $\mathrm{CD} 4{ }^{-} \mathrm{CD}^{+}$cytotoxic $\mathrm{T}$ cells requires interactions with class I MHC determinants. Nature. 1988;333(6169):180-183.

25. Chen L, Flies DB. Molecular mechanisms of T cell co-stimulation and co-inhibition. Nat Rev Immunol. 2013;13(4):227-242.

26. Sharpe AH. Mechanisms of costimulation. Immunol Rev. 2009;229(1):5-11.

27. Harding FA, et al. CD28-mediated signalling co-stimulates murine $\mathrm{T}$ cells and prevents induction of anergy in T-cell clones. Nature. 1992;356(6370):607-609.

28. Lenschow DJ, et al. Long-term survival of xenogeneic pancreatic islet grafts induced by CTLA4lg. Science. 1992;257(5071):789-792.

29. Amarante-Mendes GP, et al. Pattern recognition receptors and the host cell death molecular machinery. Front Immunol. 2018;9:2379.

30. Esensten JH, et al. CD28 costimulation: from mechanism to therapy. Immunity. 2016;44(5):973-988.

31. Zhang N, Bevan MJ. CD8(+) T cells: foot soldiers of the immune system. Immunity. 2011;35(2):161-168.

32. Schwartz RH. Historical overview of immunological tolerance. Cold Spring Harb Perspect Biol. 2012;4(4):a006908.

33. Matzinger $P$. The danger model: a renewed sense of self. Science. 2002;296(5566):301-305.

34. Palmer E. Negative selection-clearing out the bad apples from the T-cell repertoire. Nat Rev Immunol. 2003;3(5):383-391.

35. Spetz J, et al. T cells and regulated cell death: kill or be killed. Int Rev Cell Mol Biol. 2019;342:27-71.

36. Schildberg FA, et al. Coinhibitory pathways in the B7-CD28 ligand-receptor family. Immunity. 2016;44(5):955-972.

37. Jenkins MK, et al. Inhibition of antigen-specific proliferation of type 1 murine $\mathrm{T}$ cell clones after stimulation with immobilized anti-CD3 monoclonal antibody. J Immunol. 1990;144(1):16-22.

38. Asano M, et al. Autoimmune disease as a consequence of developmental abnormality of a T cell subpopulation. J Exp Med. 1996;184(2):387-396.

39. Sharma P, Allison JP. The future of immune checkpoint therapy. Science. 2015;348(6230):56-61.

40. Collins AV, et al. The interaction properties of costimulatory molecules revisited. Immunity. 2002;17(2):201-210.

41. Engelhardt JJ, et al. CTLA-4 overexpression inhibits $\mathrm{T}$ cell responses through a CD28-B7-dependent mechanism. JImmunol. 2006;177(2):1052-1061.

42. Linsley PS, et al. Coexpression and functional cooperation of CTLA- 4 and CD28 on activated T lymphocytes. J Exp Med. 1992;176(6):1595-1604.

43. Walunas TL, et al. CTLA-4 can function as a negative regulator of $\mathrm{T}$ cell activation. Immunity. 1994;1(5):405-413.

44. Brunner MC, et al. CTLA-4-Mediated inhibition of early events of T cell proliferation. J Immunol. 1999;162(10):5813-5820.

45. Linsley PS, et al. Human B7-1 (CD80) and B7-2 (CD86) bind with similar avidities but distinct kinetics to CD28 and CTLA-4 receptors. Immunity. 1994;1(9):793-801.

46. van der Merwe PA, et al. CD80 (B7-1) binds both CD28 and CTLA-4 with a low affinity and very fast kinetics. J Exp Med. 1997;185(3):393-403.

47. Wing K, et al. CTLA-4 control over Foxp $3^{+}$regulatory $\mathrm{T}$ cell function. Science. 2008;322(5899):271-275.

48. Wing JB, et al. Human $\mathrm{FOXP}^{+}$regulatory T cell heterogeneity and function in autoimmunity and cancer. Immunity. 2019;50(2):302-316.

49. Hori S, et al. Control of regulatory T cell develop ment by the transcription factor Foxp3. Science. 2003;299(5609):1057-1061.

50. Tivol EA, et al. Loss of CTLA-4 leads to massive lymphoproliferation and fatal multiorgan tissue destruction, revealing a critical negative regulatory role of CTLA-4. Immunity. 1995;3(5):541-547.

51. Waterhouse P, et al. Lymphoproliferative disorders with early lethality in mice deficient in Ctla4. Science. 1995;270(5238):985-988.

52. Kuehn HS, et al. Immune dysregulation in human subjects with heterozygous germline mutations in CTLA4. Science. 2014;345(6204):1623-1627.

53. Schubert D, et al. Autosomal dominant immune dysregulation syndrome in humans with CTLA4 mutations. Nat Med. 2014;20(12):1410-1416.

54. Latchman Y, et al. PD-L2 is a second ligand for PD-1 and inhibits T cell activation. Nat Immunol. 2001;2(3):261-268.

55. Pardoll DM. The blockade of immune checkpoints in cancer immunotherapy. Nat Rev Cancer. 2012;12(4):252-264.

56. Eppihimer MJ, et al. Expression and regulation of the PD-L1 immunoinhibitory molecule on microvascular endothelial cells. Microcirculation. 2002;9(2):133-145.

57. Greenwald RJ, et al. The B7 family revisited. Annu Rev Immunol. 2005;23:515-548.

58. Okazaki T, et al. PD-1 immunoreceptor inhibits B cell receptor-mediated signaling by recruiting src homology 2-domain-containing tyrosine phosphatase 2 to phosphotyrosine. Proc Natl Acad Sci US A. 2001;98(24):13866-13871.

59. Nishimura H, et al. Development of lupus-like autoimmune diseases by disruption of the PD-1 gene encoding an ITIM motif-carrying immunoreceptor. Immunity. 1999;11(2):141-151.

60. Waliany S, et al. Immune checkpoint inhibitor cardiotoxicity: understanding basic mechanisms and clinical characteristics and finding a cure. Annu Rev Pharmacol Toxicol. 2020;61:113-134.

61. Francisco LM, et al. PD-L1 regulates the development, maintenance, and function of induced regulatory T cells. J Exp Med. 2009;206(13):3015-3029.

62. Tan CL, et al. PD-1 restraint of regulatory $\mathrm{T}$ cell suppressive activity is critical for immune tolerance. JExp Med. 2021;218(1):e20182232.

63. Triebel F, et al. LAG-3, a novel lymphocyte activation gene closely related to CD4. JExp Med. 1990;171(5):1393-1405.

64. Anderson AC, et al. Lag-3, Tim-3, and TIGIT: co-inhibitory receptors with specialized 
functions in immune regulation. Immunity. 2016;44(5):989-1004.

65. Melero I, et al. Immunostimulatory monoclona antibodies for cancer therapy. Nat Rev Cancer. 2007;7(2):95-106.

66. Han X, Vesely MD. Stimulating T cells against cancer with agonist immunostimulatory monoclonal antibodies. Int Rev Cell Mol Biol. 2019;342:1-25.

67. Fucikova J, et al. TIM-3 dictates functional orientation of the immune infiltrate in ovarian cancer. Clin Cancer Res. 2019;25(15):4820-4831.

68. Andrews LP, et al. Inhibitory receptors and ligands beyond PD-1, PD-L1, and CTLA-4: breakthroughs or backups. Nat Immunol. 2019;20(11):1425-1434.

69. Koyama S, et al. Adaptive resistance to therapeutic PD-1 blockade is associated with upregulation of alternative immune checkpoints. Nat Commun. 2016;7:10501.

70. Leach DR, et al. Enhancement of antitumor immunity by CTLA-4 blockade. Science. 1996;271(5256):1734-1736.

71. Dong H, et al. Tumor-associated B7-H1 promotes T-cell apoptosis: a potential mechanism of immune evasion. Nat Med. 2002;8(8):793-800.

72. Iwai Y, et al. Involvement of PD-L1 on tumor cells in the escape from host immune system and tumor immunotherapy by PD-L1 blockade. Proc Natl Acad Sci U S A . 2002;99(19):12293-12297.

73. Juneja VR, et al. PD-L1 on tumor cells is sufficient for immune evasion in immunogenic tumors and inhibits CD8 T cell cytotoxicity. J Exp Med. 2017;214(4):895-904.

74. Hirano F, et al. Blockade of B7-H1 and PD-1 by monoclonal antibodies potentiates cancer therapeutic immunity. Cancer Res. 2005;65(3):1089-1096.

75. Boussiotis VA. Molecular and biochemical aspects of the PD-1 checkpoint pathway. N Engl JMed. 2016;375(18):1767-1778.

76. Hodi FS, et al. Improved survival with ipilimumab in patients with metastatic melanoma. $N$ Engl JMed. 2010;363(8):711-723.

77. Topalian SL, et al. Safety, activity, and immune correlates of anti-PD-1 antibody in cancer. $N$ Engl JMed. 2012;366(26):2443-2454

78. Brahmer JR, et al. Safety and activity of anti-PD-L1 antibody in patients with advanced cancer. N Engl J Med. 2012;366(26):2455-2465.

79. Robert C, et al. Pembrolizumab vs ipilimumab in advanced melanoma. N Engl J Med. 2015;372(26):2521-2532.

80. Mangan BL, et al. Evolving insights into the mechanisms of toxicity associated with immune checkpoint inhibitor therapy. Br J Clin Pharmacol. 2020;86(9):1778-1789.

81. Johnson DB, et al. Immune checkpoint inhibitor toxicities: systems-based approaches to improve patient care and research. Lancet Oncol. 2020;21(8):e398-e404.

82. Johnson DB, et al. Immune checkpoint inhibitor toxicity in 2018. JAMA. 2018;320(16):1702-1703.

83. Larkin J, et al. Combined nivolumab and ipilimumab or monotherapy in untreated melanoma. N Engl J Med. 2015;373(1):23-34.

84. Deutsch E, et al. Optimising efficacy and reducing toxicity of anticancer radioimmunotherapy.
Lancet Oncol. 2019;20(8):e452-e463.

85. Li W, et al. Vascular and metabolic implications of novel targeted cancer therapies: focus on kinase inhibitors. J Am Coll Cardiol. 2015;66(10):1160-1178

86. Bellinger AM, et al. Cardio-oncology: how new targeted cancer therapies and precision medicine can inform cardiovascular discovery. Circulation. 2015;132(23):2248-2258.

87. Sheng CC, et al. 21st century cardio-oncology: identifying cardiac safety signals in the era of personalized medicine. JACC Basic Transl Sci. 2016;1(5):386-398.

88. Uraizee I, et al. Reversible cardiomyopathy associated with sunitinib and sorafenib. $N$ Engl J Med 2011;365(17):1649-1650.

89. Petroni G, et al. Immunomodulation by targeted anticancer agents. Cancer Cell. 2020;S15356108(20):30601-30602.

90. Shah KP, et al. Demographic factors associated with toxicity in patients treated with antiprogrammed cell death-1 therapy. Cancer Immunol Res. 2020;8(7):851-855.

91. Eggermont AMM, et al. Adjuvant pembrolizumab vs placebo in resected stage III melanoma. $N$ Engl JMed. 2018;378(19):1789-1801.

92. Herrmann J. Adverse cardiac effects of cancer therapies: cardiotoxicity and arrhythmia. Nat Rev Cardiol. 2020;17(8):474-502.

93. Moey MYY, et al. Increased reporting of fatal pneumonitis associated with immune checkpoint inhibitors: a WHO pharmacovigilance database analysis. Eur Respir J. 2020;55(6):2000038.

94. Vozy A, et al. Increased reporting of fatal hepatitis associated with immune checkpoint inhibitors. Eur J Cancer. 2019;123:112-115.

95. Johnson DB, et al. Neurologic toxicity associated with immune checkpoint inhibitors: a pharmacovigilance study. J Immunother Cancer. 2019;7(1):134.

96. Moslehi JJ, et al. Increased reporting of fatal immune checkpoint inhibitor-associated myocarditis. Lancet. 2018;391(10124):933.

97. Mahmood SS, et al. Myocarditis in patients treated with immune checkpoint inhibitors. J Am Coll Cardiol. 2018;71(16):1755-1764.

98. Escudier M, et al. Clinical features, management, and outcomes of immune checkpoint inhibitor-related cardiotoxicity. Circulation. 2017;136(21):2085-2087.

99. Ederhy S, et al. Takotsubo-like syndrome in cancer patients treated with immune checkpoint inhibitors. JACC Cardiovasc Imaging. 2018;11(8):1187-1190.

100.Anquetil C, et al. Immune checkpoint inhibitor-associated myositis: expanding the spectrum of cardiac complications of the immunotherapy revolution. Circulation. 2018;138(7):743-745.

101.Allenbach Y, et al. Immune checkpoint inhibitor-induced myositis, the earliest and most lethal complication among rheumatic and musculoskeletal toxicities. Autoimmun Rev. 2020;19(8):102586

102. Norwood TG, et al. Smoldering myocarditis following immune checkpoint blockade. JImmunother Cancer. 2017;5(1):91.

103. Moslehi J. The cardiovascular perils of cancer survivorship. N Engl JMed. 2013;368(11):1055-1056.
104.Bonaca MP, et al. Myocarditis in the setting of cancer therapeutics: proposed case definitions for emerging clinical syndromes in cardiooncology. Circulation. 2019;140(2):80-91.

105. Caforio ALP, et al. Diagnosis and management of myocardial involvement in systemic immune-mediated diseases: a position statement of the European Society of Cardiology Working Group on Myocardial and Pericardial Disease. Eur Heart J. 2017;38(35):2649-2662.

106. Moslehi JJ, et al. Fulminant myocarditis: evolving diagnosis, evolving biology, evolving prognosis. JAm Coll Cardiol. 2019;74(3):312-314.

107. Aretz HT, et al. Myocarditis. A histopathologic definition and classification. Am J Cardiovasc Pathol. 1987;1(1):3-14.

108.Baughman KL. Diagnosis of myocarditis: death of Dallas criteria. Circulation. 2006;113(4):593-595.

109. Groarke JD, et al. Cancer-drug discovery and cardiovascular surveillance. $N$ Engl J Med. 2013;369(19):1779-1781.

110. Lee Chuy K, et al. Myocarditis surveillance in patients with advanced melanoma on combination immune checkpoint inhibitor therapy: the memorial sloan kettering cancer center experience. Oncologist. 2019;24(5):e196-e197.

111. Wang DY, et al. Cardiovascular toxicities associated with cancer immunotherapies. Curr Cardiol Rep. 2017;19(3):21.

112. Moslehi JJ. Cardiovascular toxic effects of targeted cancer therapies. N Engl JMed. 2016;375(15):1457-1467.

113. Neilan TG, et al. Myocarditis associated with immune checkpoint inhibitors: an expert consensus on data gaps and a call to action. Oncologist. 2018;23(8):874-878.

114. Ganatra S, Neilan TG. Immune checkpoint inhibitor-associated myocarditis. Oncologist. 2018;23(8):879-886.

115. Brahmer JR, et al. Management of immunerelated adverse events in patients treated with immune checkpoint inhibitor therapy: American Society of Clinical Oncology clinical practice guideline. JClin Oncol. 2018;36(17):1714-1768.

116. Puzanov I, et al. Managing toxicities associated with immune checkpoint inhibitors: consensus recommendations from the Society for Immunotherapy of Cancer (SITC) Toxicity Management Working Group. J Immunother Cancer. 2017;5(1):95.

117. Zhang L, et al. Major adverse cardiovascular events and the timing and dose of corticosteroids in immune checkpoint inhibitor-associated myocarditis. Circulation. 2020;141(24):2031-2034.

118. Mahmood SS, et al. Myocarditis with tremelimumab plus durvalumab combination therapy for endometrial cancer: a case report. Gynecol Oncol Rep. 2018;25:74-77.

119. Arangalage D, et al. Survival after fulminant myocarditis induced by immune-checkpoint inhibitors. Ann Intern Med. 2017;167(9):683-684.

120. Esfahani $\mathrm{K}$, et al. Alemtuzumab for immunerelated myocarditis due to PD-1 therapy. $\mathrm{NEnglJ}$ Med. 2019;380(24):2375-2376.

121. Salem JE, et al. Abatacept for severe immune checkpoint inhibitor-associated myocarditis. 
N Engl J Med. 2019;380(24):2377-2379.

122. Tay RY, et al. Successful use of equine antithymocyte globulin (ATGAM) for fulminant myocarditis secondary to nivolumab therapy. Br JCancer. 2017;117(7):921-924.

123. Geraud A, et al. Clinical pharmacology and interplay of immune checkpoint agents: a yinyang balance. Annu Rev Pharmacol Toxicol. 2021;61:85-112.

124. Linsley PS, et al. Immunosuppression in vivo by a soluble form of the CTLA- $4 \mathrm{~T}$ cell activation molecule. Science. 1992;257(5071):792-795.

125. Wei SC, et al. A genetic mouse model recapitulates immune checkpoint inhibitor-associated myocarditis and supports a mechanism-based therapeutic intervention [published online December 1, 2020]. Cancer Discov. https://doi. org/10.1158/2159-8290.CD-20-0856.

126. Kociol RD, et al. Recognition and initial management of fulminant myocarditis: a scientific statement from the American Heart Association. Circulation. 2020;141(6):e69-e92.

127. Hoos A. Development of immuno-oncology drugs - from CTLA4 to PD1 to the next generations. Nat Rev Drug Discov. 2016;15(4):235-247.

128. June $\mathrm{CH}$, Sadelain M. Chimeric antigen receptor therapy. N Engl JMed. 2018;379(1):64-73.

129. Jackson HJ, et al. Driving CAR T-cells forward. Nat Rev Clin Oncol. 2016;13(6):370-383.

130. Fielding AK, et al. Outcome of 609 adults after relapse of acute lymphoblastic leukemia (ALL); an MRC UKALL12/ECOG 2993 study. Blood. 2007;109(3):944-950.

131. Grupp SA, et al. Chimeric antigen receptormodified $\mathrm{T}$ cells for acute lymphoid leukemia. N Engl J Med. 2013;368(16):1509-1518.

132. Maude SL, et al. Tisagenlecleucel in children and young adults with B-cell lymphoblastic leukemia. N Engl J Med. 2018;378(5):439-448.

133. Linette GP, et al. Cardiovascular toxicity and titin cross-reactivity of affinity-enhanced $\mathrm{T}$ cells in myeloma and melanoma. Blood. 2013;122(6):863-871.

134. Cameron BJ, et al. Identification of a Titinderived HLA-A1-presented peptide as a crossreactive target for engineered MAGE A3-directed T cells. Sci Transl Med.2013;5(197):197ra03.

135. Pollack A, et al. Viral myocarditis-diagnosis, treatment options, and current controversies. Nat Rev Cardiol. 2015;12(11):670-680.

136. Grabie N, et al. T cell checkpoint regulators in the heart. Cardiovasc Res. 2019;115(5):869-877.

137. Swirski FK, Nahrendorf M. Cardioimmunology: the immune system in cardiac homeostasis and disease. Nat Rev Immunol. 2018;18(12):733-744.

138. Grabie N, et al. Endothelial programmed death-1 ligand 1 (PD-L1) regulates $\mathrm{CD} 8^{+} \mathrm{T}$-cell mediated injury in the heart. Circulation. 2007;116(18):2062-2071.

139. Kania G, et al. Innate signaling promotes formation of regulatory nitric oxide-producing dendritic cells limiting T-cell expansion in experimental autoimmune myocarditis. Circulation. 2013;127(23):2285-2294.

140. Nishimura H, et al. Autoimmune dilated cardiomyopathy in $\mathrm{PD}-1$ receptor-deficient mice.
Science. 2001;291(5502):319-322.

141. Okazaki T, et al. Autoantibodies against cardiac troponin I are responsible for dilated cardiomyopathy in PD-1-deficient mice. Nat Med. 2003;9(12):1477-1483.

142. Tarrio ML, et al. PD-1 protects against inflammation and myocyte damage in T cell-mediated myocarditis. JImmunol. 2012;188(10):4876-4884.

143. Keir ME, et al. Tissue expression of PD-L1 mediates peripheral T cell tolerance. J Exp Med. 2006;203(4):883-895.

144.Gil-Cruz C, et al. Microbiota-derived peptide mimics drive lethal inflammatory cardiomyopathy. Science. 2019;366(6467):881-886.

145. Nindl V, et al. Cooperation of Th1 and Th17 cells determines transition from autoimmune myocarditis to dilated cardiomyopathy. Eur J Immunol. 2012;42(9):2311-2321.

146.Lucas JA, et al. Programmed death ligand 1 regulates a critical checkpoint for autoimmune myocarditis and pneumonitis in MRL mice. J Immunol. 2008;181(4):2513-2521.

147. Wang J, et al. PD-1 deficiency results in the development of fatal myocarditis in MRL mice. Int Immunol. 2010;22(6):443-452.

148. Okazaki T, et al. PD-1 and LAG-3 inhibitory coreceptors act synergistically to prevent autoimmunity in mice. J Exp Med.2011;208(2):395-407.

149. Cihakova D, Rose NR. Pathogenesis of myocarditis and dilated cardiomyopathy. Adv Immunol. 2008;99:95-114.

150. Neu N, et al. Cardiac myosin induces myocarditis in genetically predisposed mice. J Immunol. 1987;139(11):3630-3636.

151. Smith SC, Allen PM. Myosin-induced acute myocarditis is a T cell-mediated disease. J Immunol. 1991;147(7):2141-2147.

152. Donermeyer DL, et al. Myocarditis-inducing epitope of myosin binds constitutively and stably to I-Ak on antigen-presenting cells in the heart. JExp Med.1995;182(5):1291-1300.

153. Galvin JE, et al. Induction of myocarditis and valvulitis in lewis rats by different epitopes of cardiac myosin and its implications in rheumatic carditis. Am J Pathol. 2002;160(1):297-306.

154. Kodama M, et al. A novel experimental model of giant cell myocarditis induced in rats by immunization with cardiac myosin fraction. Clin Immunol Immunopathol. 1990;57(2):250-262.

155. Myers JM, et al. Cardiac myosin-Th17 responses promote heart failure in human myocarditis. JCI Insight. 2016;1(9):85851.

156. Li Y, et al. Mimicry and antibody-mediated cell signaling in autoimmune myocarditis. J Immunol. 2006;177(11):8234-8240.

157. Stavrakis S, et al. Activating autoantibodies to the beta-1 adrenergic and $\mathrm{M} 2$ muscarinic receptors facilitate atrial fibrillation in patients with Graves' hyperthyroidism. J Am Coll Cardiol. 2009;54(14):1309-1316.

158. Mascaro-Blanco A, et al. Consequences of unlocking the cardiac myosin molecule in human myocarditis and cardiomyopathies. Autoimmunity. 2008;41(6):442-453.

159. Zhang P, et al. Cutting edge: cardiac myosin activates innate immune responses through
TLRs. J Immunol. 2009;183(1):27-31.

160.Krisher K, Cunningham MW. Myosin: a link between streptococci and heart. Science. 1985;227(4685):413-415.

161. Cunningham MW, et al. Cytotoxic and viral neutralizing antibodies crossreact with streptococcal $\mathrm{M}$ protein, enteroviruses, and human cardiac myosin. Proc Natl Acad Sci U S A. 1992;89(4):1320-1324.

162. Huber SA, et al. Alterations in major histocompatibility complex association of myocarditis induced by coxsackievirus B3 mutants selected with monoclonal antibodies to group A streptococci. Proc Natl Acad Sci U S A. 1994;91(12):5543-5547.

163. Massilamany C, et al. Relevance of molecular mimicry in the mediation of infectious myocarditis. J Cardiovasc Transl Res. 2014;7(2):165-171.

164. Morgan RA, et al. Cancer regression and neurological toxicity following anti-MAGE-A3 TCR gene therapy. JImmunother. 2013;36(2):133-151.

165.Zhao $\mathrm{Y}$, et al. High-affinity TCRs generated by phage display provide $\mathrm{CD} 4^{+} \mathrm{T}$ cells with the ability to recognize and kill tumor cell lines. J Immunol. 2007;179(9):5845-5854.

166. Lv H, et al. Impaired thymic tolerance to $\alpha$-myosin directs autoimmunity to the heart in mice and humans. JClin Invest. 2011;121(4):1561-1573.

167. Ammirati E, et al. Management of acute myocarditis and chronic inflammatory cardiomyopathy: an expert consensus document. Circ Heart Fail. 2020;13(11):e007405.

168. Martinez-Lostao L, et al. How do cytotoxic lymphocytes kill cancer cells? Clin Cancer Res. 2015;21(22):5047-5056.

169.Del Re DP, et al. Fundamental mechanisms of regulated cell death and implications for heart disease. Physiol Rev. 2019;99(4):1765-1817.

170.Galluzzi L, et al. Molecular mechanisms of cell death: recommendations of the Nomenclature Committee on Cell Death 2018. Cell Death Differ. 2018;25(3):486-541.

171. Voskoboinik I, et al. Perforin and granzymes: function, dysfunction and human pathology. Nat Rev Immunol. 2015;15(6):388-400.

172. Wang $\mathrm{W}$, et al. $\mathrm{CD} 8^{+} \mathrm{T}$ cells regulate tumour ferroptosis during cancer immunotherapy. Nature. 2019;569(7755):270-274.

173. Reddy J, et al. Autoimmunity in viral myocarditis. Curr Opin Rheumatol. 2013;25(4):502-508.

174. Alvi RM, et al. Cardiovascular events among adults treated with chimeric antigen receptor T-Cells (CAR-T). JAm Coll Cardiol. 2019;74(25):3099-3108.

175. Salem JE, et al. Cardiac events associated with chimeric antigen receptor T-cells (CAR-T): a VigiBase perspective. J Am Coll Cardiol. 2020;75(19):2521-2523.

176. Ganatra S, et al. Chimeric antigen receptor T-cell therapy-associated cardiomyopathy in patients with refractory or relapsed non-Hodgkin lymphoma. Circulation. 2020;142(17):1687-1690.

177. Lefebvre B, et al. Cardiovascular effects of CAR T cell therapy: a retrospective study. JACC CardioOncol.2020;2(2):193-203. 